\title{
À propos de la tradition conradienne du Cour des ténèbres comme archive internationale
}

\section{Pierre Halen}

\section{OpenEdition}

\section{Journals}

Édition électronique

URL : https://journals.openedition.org/coma/2953

DOI : 10.4000/coma.2953

ISSN : 2275-1742

Éditeur

Institut des textes \& manuscrits modernes (ITEM)

\section{Référence électronique}

Pierre Halen, «À propos de la tradition conradienne du Cœur des ténèbres comme archive internationale », Continents manuscrits [En ligne], 11 | 2018, mis en ligne le 15 octobre 2018, consulté le 12 janvier 2023. URL : http://journals.openedition.org/coma/2953; DOI : https://doi.org/10.4000/ coma.2953

Ce document a été généré automatiquement le 12 janvier 2023.

\section{cc)}

Creative Commons - Attribution - Pas d'Utilisation Commerciale - Pas de Modification 4.0 International - CC BY-NC-ND 4.0

https://creativecommons.org/licenses/by-nc-nd/4.0/ 


\title{
À propos de la tradition conradienne du Cœur des ténèbres comme archive internationale
}

\author{
Pierre Halen
}

1 La référence - explicite ou implicite, consciente ou inconsciente - à Heart of Darkness [Le Cœur des ténèbres] ne cesse, on le sait, de hanter les productions culturelles. Depuis quand ? C'est plus difficile à déterminer, car il s'agit d'une «fortune », comme disaient autrefois les comparatistes, qui s'est lentement accumulée avec le temps. Elle semble bien, toutefois, avoir connu une accélération progressive après la Seconde Guerre mondiale, et un essor considérable à l'époque post-coloniale ${ }^{1}$. On la retrouve ainsi dans le discours qui s'énonce à propos de l'Afrique centrale comme dans les essais et dans les œuvres esthétiques qui s'en réclament, qui en traitent ou qui, de manière un peu plus lâche, l'englobent parmi d'autres objets, par exemple dans le cadre d'une réflexion concernant les génocides ${ }^{2}$. Il ne sera pas question ci-dessous, on le comprendra, d'un inventaire de ces productions ou de ces discours, opération que le caractère diffus, indirect ou analogique de la référence à la nouvelle de Joseph Conrad rend impossible, sauf à prendre en compte des critères limitatifs (ne considérer, par exemple, que tel genre discursif, ou telle explicitation verbale).

2 Nous reprendrons ici, en espérant apporter un certain éclairage au moins à la discussion commune, un certain nombre d'hypothèses déjà formulées ${ }^{3}$. Cette fois, partons du concept contemporain d'archive, au sens d'un discours archétypique, matriciel ou structurant, en référence seulement à un document ancien qu'il ne s'agit pas de conserver ni d'étudier, mais de réinterpréter, voire de reconfigurer, quitte, éventuellement, à lui faire violence ${ }^{4}$. Coeur des ténèbres, parce qu'il innerve, informe et en quelque sorte nourrit l'imaginaire depuis plus d'un siècle, pourrait certes aussi être considéré comme un mythe, ou encore comme un schème, mais le concept d'archive nous évitera d'entrer dans un débat spécialisé avec les théoriciens des genres littéraires ou avec les philosophes. 
3 Une archive, donc, mais une archive de quoi ? C'est la question essentielle, mais la réponse ne va nullement de soi. Pour certains, c'est une archive de l'État Indépendant du Congo (EIC), un témoignage ou une réflexion narrative concernant cette époque, donc concernant l'Histoire de l'Afrique centrale en des temps marqués par diverses violences 5 . Pour d'autres, sensibles aux éléments structurels de ces violences à plus long terme, c'est une archive de la globalisation et de la dérégulation libérales dont l'EIC n'est qu'une illustration exemplaire, ou celle d'une modernité tragique, ou encore l'archive de logiques génocidaires, souvent présentées comme inhérentes au capitalisme et/ou à l'Occident. Pour d'autres enfin, c'est une archive de l'humanité même, réveillant le souvenir de mondes plus anciens, primitifs, mais à ce titre essentiels et déterminants : le cœur de l'humain, en somme sa nature véritable, révélant son "horreur" indéterminée; le récit de ces temps "obscurs" et ces "pages sombres" sont dès lors lus comme une sorte de parabole dans un discours radicalement pessimiste à propos de l'Histoire qu'on disait alors " universelle ", voire à propos de l'Être même: ne songeons qu'à la focalisation des commentateurs contemporains sur deux phrases de Kurtz: "The horror, the horror» et le fameux «Exterminate all these brutes». En somme, il y aurait ainsi une «matière » conradienne comme il y a, au théâtre, une scène mythologique thébaine : or, puisque nul ne songe à voir, par exemple, dans le personnage de Phèdre un témoignage à propos de la pratique l'inceste dans la Grèce antique, il n'y aurait pas de raison non plus pour voir dans celui de Kurtz un exemple représentatif de ce que fut historiquement l'EIC.

\section{Un contexte international}

Quoi qu'il en soit de ces interprétations, le caractère international de cette archive est une évidence, et ce, dès la première publication du récit. Certes, celle-ci n'a d'abord lieu que dans le champ littéraire anglais de la fin du XIXe siècle, où un écrivain d'origine polonaise qui n'est pas encore reconnu tente de se faire une place au soleil ; mais, dès le départ, il s'agit déjà d'une prise de position qui se fait en référence à un État qui est alors l'objet depuis plusieurs années de campagnes médiatiques et de controverses, internationales elles aussi, avec un foyer spécialement actif en Angleterre. Ajoutons que ces débats n'ont eux-mêmes lieu que dans le cadre plus général du grand feuilleton éditorial que constitue en Europe, depuis des décennies, l'exploration de l'Afrique (voir la contribution d'Andrew Griffiths). Aux controverses, les écrivains participent : comme on le sait, la publication de Heart of Darkness précède ainsi de peu, notamment, le King Leopold's soliloquy (1905) de Mark Twain et The Crime of the Congo (1909) d'Arthur Conan Doyle, écrits qui connaissent une large diffusion. On a oublié le nom des auteurs de fiction et de non-fiction qui publièrent à l'époque des livres prenant au contraire fait et cause pour l'entreprise léopoldienne, entreprise qui se présenta elle-même comme humanitaire, selon le vocabulaire d'aujourd'hui, et, dans son intitulé même, comme « internationale $»^{6}$.

5 Ce caractère international devait être rappelé ici malgré son évidence, car les reprises et les développements ultérieurs de la tradition conradienne, même s'ils n'ont plus pour contexte ce qu'on appelait à l'époque les « campagnes anti-congolaises » dirigées contre l'État léopoldien, ont néanmoins encore et toujours pour cadre des campagnes du même ordre et à propos du même référent historique. Le King Leopold's ghost : a story of greed, terror, \& heroism in colonial Africa (1998) d'Adam Hochschild, s'il n'apprenait pas 
grand-chose aux historiens, a été immédiatement traduit en français, en néerlandais, en allemand, en espagnol... et multidiffusé en divers formats et transpositions. Ici aussi, la fiction a fait mousser l'imaginaire : il y avait déjà eu un opéra intitulé Red Rubber en 1993, il y aura par exemple, à partir de 2010, la suite des albums de bande dessinée intitulés (curieusement, compte tenu de leur ambition historique) Africa dreams ${ }^{7}$; ou alors la fictionalité structurera le documentaire, comme la théatralisation judiciaire sur laquelle joue le film de Peter Bate White King, Red Rubber, Black Death (2004). Dans ce cas encore, il y a eu un writing back, de la part d'historiens autorisés ou amateurs, tous en appelant à un peu d'objectivité : ce n'est pas le lieu d'entrer dans les détails de ces débats ici, ce qui supposerait d'analyser la manière dont un jugement historien quelconque peut être ou non en phase avec une certaine attente institutionnelle et/ou médiatique ${ }^{8}$, ou quel intérêt il a de s'esthétiser pour "communiquer » (et quel en est alors le prix à payer). Contentons-nous d'observer deux faits : d'abord, la nouvelle de Conrad ne s'est pas détachée, avec le temps, de son contexte de référence (ce qui aurait été le cas s'il s'était agi d'un mythe), mais elle semble au contraire avoir informé celui-ci, c'est-à-dire déterminé en bonne part sa représentation médiatique. Ensuite, la controverse concernant l'EIC s'est peu à peu mélangée, au cours des années 2000, à celle qui concernait les responsabilités de la Belgique dans l'assassinat de Patrice Lumumba en 1961, débat qui se manifeste, dans certains lieux du Royaume mais aussi ailleurs, par une petite guerre médiatique à propos de la toponymie des espaces publics et des statues à déboulonner (ou non) ou à édifier (ou non), qui n'est pas terminée à l'heure actuelle.

Ceci pose finalement la question de savoir si Coeur des ténèbres n'est pas une pièce essentielle dans les références culturelles d'une mouvance transnationale qui se met en place à la fin du XIX ${ }^{e}$ siècle ; intellectuelle et bourgeoise, lettrée en tout cas, elle semble se constituer dans le contexte historique de l'impérialisme colonial comme phénomène mondial lié à l'expansion de la presse, et trouver l'une de ses justifications dans la dénonciation de ses scandales. Ces derniers constitueraient en d'autres termes un sujet de conversation commun pour cette communauté émergente, sensibilisée à la nécessité d'agiter les chiffons rouges de la conscience humanitaire à l'encontre des intérêts strictement nationaux qui sont déterminants dans l'impérialisme européen de l'époque; de même, plus récemment, contre les intérêts politico-économiques des puissances libérales de la mondialisation (ainsi, dans certains films d'Hubert Sauper, la tradition conradienne est mise au service de dénonciations d'horreurs à la fois humanitaires et écologiques). Cela expliquerait peut-être pourquoi le nom de Kurtz ne renvoie à rien de typiquement "belge", mais suggère tout au plus une vague germanité , de même d'ailleurs que le nom de plume de Conrad lui-même; et, de même, pourquoi le personnage de l'invraisemblable voyageur russo-hollando-anglais (fig. 1) en habit d'arlequin que Conrad a imaginé pour Coeur des ténèbres a pris ensuite une telle importance, notamment dans les deux adaptations cinématographiques de cœur des ténèbres. 
Figure 1. Une figuration parmi d'autres du jeune voyageur mondialisé que Marlow découvre en arrivant chez Kurtz
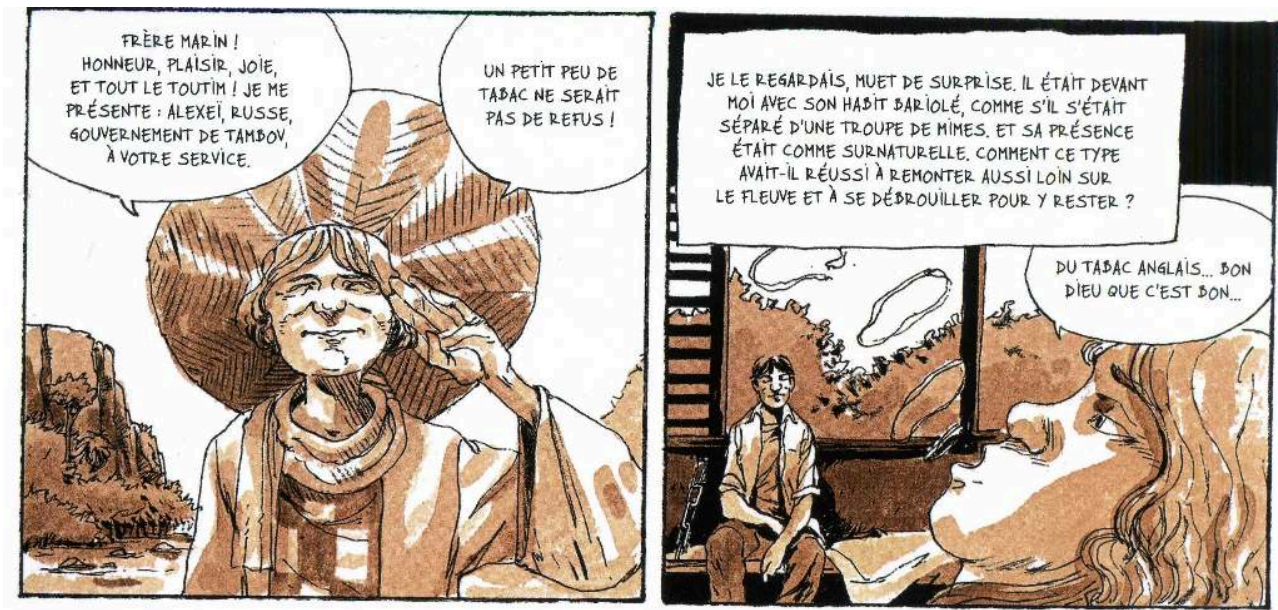

Au cœur des ténèbres, scénariste : Stéphane Miquel, dessinateur et coloriste : Loïc Godart, Toulon, Soleil productions, coll. « Noctambule», 2014, 104 p. ; 28,3 cm ; p. 71.

Ce cadre étant posé, on trouvera ci-dessous quelques rappels à propos du genre littéraire problématique de Cœur des ténèbres, du contexte dans lequel celui-ci a été conçu, mais aussi de sa postérité, ce qui, dans un deuxième temps, nous permettra de cerner, d'une manière globale, la tradition conradienne, car tradition il y a (au sens général de «transmission de génération en génération à l'aide de la parole ou de l'exemple ", voire au sens religieux, formulé ainsi par Émile Littré : «Transmission de faits historiques, de doctrines religieuses, de légendes, etc. d'âge en âge par voie orale et sans preuve authentique et écrite $\left.{ }^{10} »\right)$. Dans un troisième temps, nous nous intéresserons aux composantes narratives et thématiques, aux invariants et aux variantes dont la «fortune » se mesure au nombre de reprises ultérieures. Tout cela nous permettra de voir se dégager une sorte de matrice imaginaire, que nous pourrons situer dans le cadre général d'un exotisme critique, où l'on verra que les aspects formels, thématiques et idéologiques sont étroitement liés.

\section{Genres et contextes}

Ce n'est pas le lieu ici d'étudier la position d'entrant qu'occupe Conrad au moment où il publie Heart of Darkness dans le Blackwood Magazine ${ }^{11}$. Du point de vue biographique, rappelons néanmoins que ce récit peut être lu aussi comme un règlement de compte personnel après une expérience congolaise ressentie comme décevante, voire humiliante. Par ailleurs, du point de vue de l'actualité médiatique, nous avons déjà signalé qu'elle constitue forcément une prise de position très "anglaise » dans les débats assez violents de l'époque - leur violence croîtra encore jusqu'en 1906 - à propos du Congo léopoldien. Ces débats sont également alimentés en France, en Allemagne et dans divers pays occidentaux, dont les États-Unis ; si le Royaume-Uni et la France sont les pays où la controverse est la plus nourrie, il s'agit aussi des deux puissances coloniales les plus directement intéressées par une éventuelle disparition de l'EIC, ou à tout le moins par une reprise de contrôle sur celui-ci et/ou une limitation de son expansion territoriale ${ }^{12}$ (ceci, bien entendu, ne signifie nullement que Morel, Casement et consorts ne soient pas des consciences sincères et désintéressées). 
9 Par ailleurs, comme nous l'avons déjà suggéré, les nombreuses traductions et la diffusion internationale des écrits des «explorateurs» de l'Afrique centrale, ceux de Livingstone et de Stanley en particulier, ont dès cette époque largement contribué à situer les territoires concernés dans une position de tiers imaginaire commun aux pays occidentaux; c'est le résultat d'une abondante production dans la presse périodique comme en librairie, où l'humanitarisme anti-esclavagiste et la fascination pour les sources du Nil ont le même statut fonctionnel que les scandales liés aux violences rapportées, qu'il s'agisse du Red Rubber ou de l'«affaire Stokes ». Les uns comme les autres font partie d'une conversation européenne commune, dont l'objet est l'expansion européenne, et plus fondamentalement la nouvelle configuration d'un monde que les bateaux à vapeur, le télégraphe et la presse quotidienne commencent à unifier pratiquement, non sans procurer à l'auto-identification du sujet européen le sentiment qu'il aurait une " mission » historique, laquelle mission ne se réduirait pas à la justification d'une conquête territoriale. Même si, pour des motifs personnels et familiaux notamment, Jozef Konrad Korzeniowski a certainement un contentieux avec l'idée d'Empire (c'est l'incarnation russe de cette idée qui en a fait un orphelin, puis un exilé), il donne le sentiment à son lecteur anglais du temps qu'il s'attaque moins à l'impérialisme en général, encore moins au colonialisme britannique, qu'à cet impérialisme-là, minable, dévoyé, indigne de la haute mission qu'il aurait dû, noblement et surtout efficacement, transformer en actes et en réalisations à la hauteur de ses ambitions.

10 Cette position nettement marquée dans un champ national et dans un temps déterminé n'empêche dès lors nullement que l'œuvre conradienne, dans son ensemble, nous apparaisse aujourd'hui à juste titre comme une œuvre-monde, la première peut-être dans l'histoire des littératures; c'est l'effet des voyages fictionnalisés (qui font qu'elle ne s'insère que de biais dans la littérature coloniale) et de la multiplicité des lieux planétaires qui y sont évoqués, mais sans doute aussi de thématiques particulières, sur le double fond de la conscience transnationale émergente dont nous avons déjà parlé, et sans doute d'une mélancolie diffuse, qui lui est propre. À cet égard, ce n'est bien sûr pas sans motif que les commentateurs ont vu dans la figure très post-coloniale de Corto Maltese, mise en fiction par Hugo Pratt à partir de 1965, un héritier de Marlow.

11 C'est ce qui complique aussi l'attribution d'un genre littéraire, ou même seulement d'un genre textuel, à Couur des ténèbres. Le plus simple est évidemment, sur la base de sa première publication en revue puis dans un recueil de trois récits, de la qualifier de longue nouvelle : on ne court pas le risque de se tromper. Cela dit, on sait qu'il y a bien plus à répondre à cette question et que le texte se signale, à cet égard aussi, par son ambivalence. Passons sur la tentation d'y voir un roman, ce que le flou de la catégorie autorise et que l'opportunité commerciale favorise (on en fait ainsi un livre en soi). Il est plus intéressant d'observer comme le texte joue en même temps sur les genres de l'autobiographie et du document authentique, mais aussi du conte (qui éclaire sa structure enchâssée), et notamment du conte initiatique, voire du mythe, avec une forte teneur religieuse ou, à tout le moins, spirituelle.

12 Au-delà de cette ambivalence générique, on rappellera aussi son ambivalence idéologique : Coeur des ténèbres est très souvent invoqué à l'appui d'une dénonciation des méfaits du colonialisme, mais on a fait observer à juste titre, d'abord, sa dimension raciste $^{13}$, surtout sensible dans l'effacement quasi total des Congolais comme sujets de l'Histoire et dans leur représentation tantôt anonyme, tantôt « sauvage ». Par ailleurs, 
comme nous l'avons suggéré plus haut, ce n'est pas tant la colonisation qui y fait l'objet d'un portrait-charge, que la manière indigne dont les "pèlerins sans foi » de l'EIC, inefficaces, tentent en vain d'en réaliser le programme historique : la nouvelle diffuse donc bien, en creux ou par son absence, la représentation coloniale de l'« homme d'énergie en pays neuf ", caractérisé par la restraint qui fait tellement défaut aux nonAnglais.

Le troisième niveau auquel il faut situer l'œuvre est sans doute le plus important, dès lors qu'on la considère pour ce qu'elle est: une œuvre littéraire qui s'inscrit dans un champ fortement autonomisé déjà. De ce point de vue, la nouvelle ne fait pas mystère (la plume de Conrad est même lourdement insistante) de ses affinités avec la sensibilité «fin-de-siècle » et avec la pensée critique du temps, pensée dominée dans ses avantgardes par l'influence du symbolisme, et notamment de Schopenhauer. C'est peu dire qu'on ne voit pas grand-chose dans Couur des ténèbres, tant les lumières sont faibles et vacillantes, jusqu'à la dernière flamme qui s'éteint au moment de la mort de Kurtz, et tant la nuit le dispute, le jour, au brouillard et à la brume. L'adaptation de Coppola, Apocalypse Now (1979), exploite magistralement le potentiel à la fois esthétique et moral de ce champ sémantique, alors que celle de Nicolas Roeg, Heart of Darkness (1993), pourtant plus fidèle au récit initial, transpose assez peu et assez maladroitement cet aspect: c'est que le film de Nicolas Roeg est, formellement, d'inspiration plus naturaliste comme il est, idéologiquement, d'orientation plus dénonciatrice ${ }^{14}$.

En somme, si le récit de Conrad est sans doute une œuvre de "résistance », comme toute œuvre moderne, à quoi résiste-t-elle ? Au-delà du règlement de compte personnel et du contexte de ce qu'on appelait alors la "campagne anti-congolaise ", c'est à la doxa triomphante du temps qu'elle s'oppose avec le plus de force : une doxa à la fois coloniale, progressiste et naturaliste, confiante dans le progrès de l'humanité planétaire qui, en s'appuyant sur les bienfaits conjugués de la science, de la technique et du commerce libéral, devait la conduire à l'unification du genre humain et au bonheur de tous dans un confort partagé, y compris du point de vue juridique ${ }^{15}$. Nous avons du mal à reconstituer ce qu'a représenté, à la fin du XIX ${ }^{e}$ siècle, l'unité de cette triade, d'abord en raison de ce que le $\mathrm{xx}^{\mathrm{e}}$ siècle nous a appris au sujet des redoutables capacités de l'espèce humaine, non seulement à produire des inégalités, mais aussi à s'autodétruire comme à détruire son environnement naturel, jusqu'à menacer consciemment, mais sans vraiment réagir, la survie même des générations ultérieures. Nous avons du mal aussi à la reconstituer parce que l'anticolonialisme a sérieusement battu en brèche l'idée d'universel. Il faut cependant essayer de se la représenter, notamment parce qu'elle continue de sévir, de fait, dans une bonne partie des représentations et donc des réalités contemporaines, qui investissent ingénument des fortunes dans des opérations dévastatrices au nom de la « croissance » ou seulement au nom d'intérêts privés, mais aussi parce qu'elle seule permet de voir, a contrario, le type de modernité auquel s'oppose Cour des ténèbres (fig. 1). C'est déjà celle à laquelle s'attaquait Flaubert, dès 1857, à la fin de Madame Bovary, en dénonçant le «train d'enfer ", c'est-à-dire le triomphe totalitaire, du pharmacien Homais, modèle du cuistre et grand zélateur du progrès scientifique; pour nous limiter à un second exemple très connu, c'est aussi cette modernité du progrès technique que récuse un Maeterlinck lorsqu'il représente au théâtre, en 1893, une Mélisande perdue dans la nuit de l'univers. La triade en question n'est ni à gauche ni à droite de l'échiquier politique, ou plutôt elle est une sorte de racine commune aux deux façons de concevoir le " développement ", 
et c'est précisément ce qui explique la difficulté de faire servir le texte conradien, qui est d'abord une œuvre d'artiste, à un parti seulement, colonialiste ou anti-colonialiste singulièrement : il vise un élément civilisationnel plus profond, plus central ou plus profondément enfoui dans l'identité occidentale: la conviction qu'il y a un «sens de l'Histoire ». Inversement, on voit dès lors aussi pourquoi ce serait également mal lire la nouvelle que de n'y voir que des ambitions esthétiques ou littéraires.

\section{La fortune de Heart of Darkness (quelques réalisations)}

Dès 1992, Bernard Piniau (op.cit.) avait clairement reconstitué quelques repères essentiels dans la tradition conradienne au sein des littératures européennes, tel qu'on pouvait l'appréhender à l'époque à partir d'un comparatisme qui s'intéressait essentiellement aux grandes œuvres les plus connues, romanesques en l'occurrence. En particulier, il montrait la présence de ces représentations héritées de Conrad dans les œuvres de Graham Greene - principalement A Burnt-Out Case (1960) -, d'Alberto Moravia - A quale tribù appartieni ? (1972), La donna leopardo, 1991 -, plus tard de V.S. Naipaul - surtout In a Free State (1971) et A Bend in the river (1979) -, toutes œuvres très internationales quant à leur diffusion. De nombreux autres titres seraient bien sûr à mentionner également, à commencer par le Voyage au bout de la nuit de Céline (1932), bien que ce roman ne se réfère pas explicitement à l'Afrique centrale. J'ai indiqué ailleurs qu'on retrouve cette tradition conradienne dans le corpus belge, notamment dans le roman Kufa (1954) de Henri Cornélus, réputé lui aussi anti-colonialiste, et dans le corpus congolais, singulièrement dans Cannibale (1986) de Bolya, dont Silvia Riva a étudié la "rhétorique des ténèbres ${ }^{16}$ ». Nous avons vu qu'elle était présente au cinéma, et l'on peut en dire autant de la bande dessinée, surtout du secteur adulte et avantgardiste ${ }^{17}$, qui correspond au milieu socio-culturel «international» dont nous avons parlé.

Plus largement, la tradition conradienne se retrouve dans l'ensemble de ce que Marc Angenot a appelé le discours social, singulièrement dans la presse et les médias audiovisuels, singulièrement lorsqu'il est question, bien sûr, de représenter l'Afrique centrale : la démonstration en a été faite par Bernard Piniau pour les années 1960-1961. Il faut certes insister sur les conditions de production inhérentes à toute communication « de masse » : on y reprend nécessairement les données d'abord sous la forme de clichés, et nous avons vu que l'imagerie ténébreuse de Conrad s'était largement imposée, par sa puissance symbolique, corollaire de son ambivalence idéologique, dans l'imaginaire occidental et mondialisé, où elle est d'autant moins filtrée par les intellectuels que ceux-ci s'y retrouvent, non dans l'image du monde qu'ils construisent et regardent, mais en face, dans leur propre position de voyeurs mélancoliques et de dénonciateurs de ce qu'ils désignent comme étant le fait des autres. C'est pourquoi cette imagerie ténébreuse est, comme le dit l'expression, dans toutes les mémoires et fait retour presque mécaniquement, y compris dans les productions les plus éloignées des mythes et de la fiction. Nous y reviendrons à propos du symbole particulièrement évocateur que constitue le steamer.

17 Le concept de continent rétif, proposé par Luc Rasson ${ }^{18}$, éclaire en profondeur la tradition conradienne et ses ambiguïtés politiques : certes, il s'agit, chez Céline après Conrad, de l'Afrique comme continent obscur et même ténébreux, mais la référence que font ces deux auteurs à l'Afrique est à double fond, voire purement 
conventionnelle : c'est en réalité l'humanité qui est rétive à ses propres ambitions et à ses propres valeurs. Rétivité certes aberrante, contradictoire, suicidaire, mais dont les effets sont vérifiables tous les jours dans n'importe quelle gazette. Rétivité impensable, en tout cas pour le positivisme et le scientisme matérialiste; mais dicible en revanche dans le discours religieux et, certainement, racontable, dessinable, filmable dans l'art et dans la fiction, quitte à accepter de voir faire retour dans le récit ce vieux personnage : le diable, singulier ou pluriel, présent à divers titres dans Cœur des ténèbres, qui n'est pas spécialement un texte religieux.

\section{La tradition conradienne (composantes, invariants structurels)} conradienne, tradition narrative comportant des invariants structurels et des éléments récurrents, présents dans la nouvelle de Conrad mais aussi ajoutés au fil du temps par les imaginations qui s'en sont emparées et l'ont enrichie. Autant de lieux communs ou, si l'on veut, d'«idées reçues» formant une imagerie ambivalente comme celle du mythe, mais cohérente et féconde.

\section{Un premier groupe d'invariants d'ordre narratologique et fictionnel}

Il s'agit d'abord de la progression du personnage principal, Marlow, le sujet-narrateur ordinaire, vers Kurtz, l'être extraordinaire. Cette progression se fait malgré les obstacles, à partir d'un moment préalable de piétinement, et ses étapes sont marquées par l'obtention régulière de renseignements partiels, toujours liés à une sorte de fascination ambivalente caractérisant les informateurs ; celle-ci relance à chaque fois la curiosité du personnage principal, de plus en plus fasciné lui-même quoique jamais entièrement. La rencontre avec Kurtz précipite la fin de cette quête et de la fascination, sans mettre fin au trouble et aux doutes du narrateur. Un second invariant structurel, bien connu, est la structure du récit enchâssé : Marlow est d'abord présenté comme personnage à la troisième personne avant de raconter sa propre histoire. Enfin, divers personnages conradiens sont repris d'une réécriture à l'autre, avec des développements éventuellement différents à chaque fois: Kurtz et Marlow, bien sûr, la fiancée européenne ${ }^{19}$, la compagne africaine, le jeune traitant ex-russe converti à une sorte de religion kurtzienne, le pilote congolais du vapeur, mais aussi les groupes anonymes d'Africains qui montent la garde autour du personnage de Kurtz, les «pèlerins sans foi » des comptoirs coloniaux, le directeur, etc.

\section{Un deuxième groupe d'invariants relatifs à l'espace}

Les motifs liés à l'espace dessinent bien plus qu'un décor. C'est ici qu'on rencontre le fleuve, élément essentiel, avec des sous-motifs non moins fondamentaux. Le cours d'eau, dont on souligne notamment l'immensité et la puissance, est à remonter vers sa source, vers l'« intérieur ", le « cœur », qui est à la fois le noyau essentiel du continent, l'origine, mais aussi la fin, ce que Graham Greene surtout présentera comme le "bout » / «the end » dans A Burnt-out Case ${ }^{20}$. Remonter l'espace du fleuve, c'est donc remonter le temps en allant vers l'origine : d'où la présence, d'abord, de la boue originelle qui teinte

Continents manuscrits, 11 | 2018 
les eaux d'une couleur « kaki », mélangée, trouble, indistincte. Mais cette remontée est difficile et lente, puisqu'elle se heurte à des forces nécessairement supérieures, celles de la Création elle-même. D'où aussi les suggestions préhistoriques, les pachydermes et les sauriens, en particulier, étant associés dans l'imaginaire à des temps primitifs, antérieurs à la présence de l'homme et étrangers à lui. Un sous-motif particulier, celui des îlots de jacinthes d'eau arrachés à la vase et dérivant d'est en ouest, vers l'Occident et l'anéantissement final, donc, est déjà présent chez Greene mais sera surtout développé par Naipaul dans A Bend in the River (1980), l'auteur étant certes inspiré par la prolifération plus importante de la plante à l'époque post-coloniale, mais bien entendu il s'agit aussi là d'un symbole d'une dégénérescence d'un autre ordre, socio-politique au moins.

Le référent géographique fournit par ailleurs une autre imagerie, celle du dark jungle wall, le mur opaque que constitue la forêt équatoriale pour le regard, donc pour le désir de connaissance. Les couvertures de très nombreuses éditions de Heart of Darkness, dans toutes les langues, ne manquent pas d'exploiter, pertinemment d'ailleurs, ce motif essentiel, qui ne concerne pas seulement le rapport épistémologique à la nature mais aussi à l'humanité des " autres » que sont les Congolais qu'on voit parfois apparaitre et qui disparaissent ensuite dans le "mur " (aspect récurrent aussi dans An Outpost of Progress). Cette impossibilité de voir au-delà de l'espace restreint qu'on occupe soimême est redoublée par deux autres motifs: celui des "ténèbres", bien sûr, notamment nocturnes et associées à l'idée de la darkness du mur végétal; et celui des brumes et brouillards perturbant la vision et produisant le sentiment de l'irreprésentable, donc aussi de l'inopérabilité du monde. Soulignons que le mur en question est une paroi immobile, éternelle, pas un obstacle quelconque dont la technique coloniale-positiviste aurait un jour raison : dans la tradition conradienne, les choses ne sauraient changer ${ }^{21}$, sinon pour le pire.

Enfin, une troisième structure spatiale revient: l'opposition entre la jungle " primitive » et les métropoles de banquiers bourgeois, Londres et Bruxelles, opposition illustrée notamment en langage visuel par le film de Nicolas Roeg, assez fidèle à la nouvelle de ce point de vue.

Dans cet ensemble spatial, il est sans doute éclairant de faire observer ce qui n'apparaît pas : les missions religieuses, les ponts et les routes, motifs qui seront mis à l'avant-plan par les représentations (et l'action) coloniales; comme référents, certes, ils existaient peu encore à l'époque de Conrad, mais ceci n'est en rien une explication : Stanley et ses illustrateurs sont de la même époque et ne manquent pas, quant à eux, de jouer notamment sur le symbole de la route (auquel est lié le sobriquet de Boula-Matari, le casseur de pierre), route sur laquelle il fait même passer des bateaux. Autre absence non moins significative: les sociétés indigènes, en tant que groupes structurés en dehors des Européens : elles sont inexistantes, inconnaissables et inconnues, ce qui n'est pas le cas chez Stanley.

\section{Une troisième catégorie de motifs plus abstraits}

Elle réunit des éléments de langage qui sont des qualifications philosophiques et/ou religieuses. Ils abondent dans le texte même de Conrad, qui n'a pas pour rien été qualifié à la fois de symbolique et de symboliste. Contentons-nous de l'essentiel, puisque les commentateurs ont été nombreux à insister déjà sur ce point. Un champ 
sémantique majeur est celui du Mal et du Diable, associé culturellement au motif religieux des ténèbres ${ }^{22}$, lui-même se réfléchissant dans le discours "profane" de l'obscurité et de l'obscurantisme que devraient éclairer, mais que ne parviennent pas à éclairer, les «lumières » de la science conquérante, lumières ici dévoyées en torches allumant des incendies, autre motif infernal. Le Diable, dans ses différentes manifestations, est certes en collusion, de fait, avec un capitalisme débridé et même "sauvage ", mais la poéticité du texte conradien ne permet pas d'en faire un symbole monovalent qui empêcherait une lecture religieuse: Kurtz est bien plus qu'une incarnation des déviances contextuelles d'un système de gouvernance particulier. Il est ce dont l'humain est capable, peut-être sa nature profonde d'ange pervers et déchu. De là, aussi, l'ambivalence du progress, un mot qui se retrouve, avec des correspondants dans diverses langues, au carrefour entre le discours religieux (au sens de pèlerinage et d'itinéraire spirituel) et le discours techno-scientifique alors dominant (les progrès de l'humanité sous l'égide de la triade positiviste que nous avons dite). À divers égards, Marlow est un de ces sujets positivistes de bonne volonté, obligé de s'initier à un mystère qui le dépasse, parce qu'il dépasse le cadre de pensée et les valeurs alors dominantes.

On peut ranger ici, enfin, le thème apparemment plus politique et civilisationnel de l'aventurier-roi, bien étudié naguère par Gilbert Soubigou : projection fantasmatique de l'identité européenne à un moment où elle cherche à représenter sa place dans un monde qu'elle ne peut voir que comme autre (exotique, donc), c'est une figure bien connue de la fiction d'aventures coloniales, qu'illustreront Tarzan et bien d'autres personnages de productions plus légitimes, de Simenon à Malraux. Notons que la figure d'aventurier-roi qu'est ici Kurtz est, elle aussi, une figure potentiellement religieuse, s'entourant des signes de la royauté théologico-politique qu'est supposée souvent cautionner - dans le récit colonial comme dans les aventures de Tintin, ou encore dans les récits contemporains d'opérations humanitaires - le recours à la science occidentale (guérisons médicales, démonstrations savantes éblouissant les populations " primitives »). Ce n'est pourtant pas le cas chez Conrad, où Kurtz affirme sa supériorité par une sorte de nature supérieure de héros efficace, ce qui sera exploité notamment par Coppola.

\section{Du steamer}

Symbole unissant et condensant l'ensemble de ces questions, le steamer est au cœur de la représentation conradienne. En tant que "vapeur ", il symbolise toute la révolution industrielle et la puissance qui se trouve désormais entre les mains de l'entrepreneur européen pour « mettre en valeur » le monde. Rappelons que ces bateaux à vapeur sont également centraux sous la plume et dans les actes de Stanley, mais avec le sens opposé d'une confiance dans le progrès technique et ce qu'il peut apporter. Chez Conrad, le steamer - quand il fonctionne, puisqu'il faut d'abord en trouver un qui soit réparable et le remettre en ordre de marche - est toujours en peine de remonter un courant trop puissant pour lui. 
Figure 2. Une page aquarellée dédiée au steamer s'avançant dans la brume (dans le sens contraire à celui de l'histoire), pour marquer le début d'un chapitre dans Congo 40 , une fiction représentative de l'anti-colonialisme

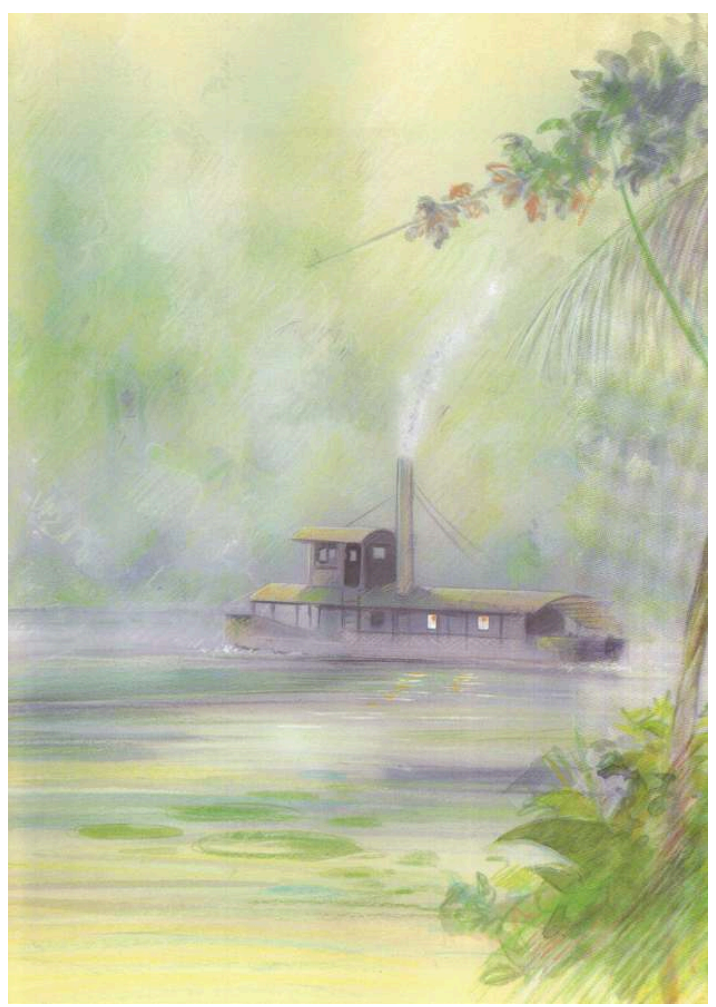

Éric Warnauts et Guy Raives, Congo 40, Tournai, Casterman, coll. « Studio (À Suivre) », 1988, 85 p. ; $29,5 \mathrm{~cm}$.

Ainsi le «progrès" n'aura-t-il jamais raison de ce qui, dans le réel, lui résiste. Contentons-nous d'un exemple, emprunté à Graham Greene, et en l'occurrence au début de A Burnt-out Case, lorsque l'ex-architecte à succès Querry se trouve sur le petit vapeur qui tente de remonter le courant pour atteindre la mission: « [...] the passenger found that he could watch, with a kind of inert content, the thick, rapid, khaki-coloured stream against which the small boat fought his way at about three knots, the engine, somewhere below the altar and the Holy Family, groaning like an exhausted animal and the big wheel churning away at the stern. A lot of effort it seemed for so slow a progress. [...] ». Cette dernière formule est particulièrement claire : quelles que soient la dépense et l'ingéniosité des hommes, la "progression » sera toujours insuffisante, et dans ces conditions, il n'est peut-être pas insensé, en désespoir de cause, de prier la Sainte Famille.

Dans la vision conradienne, le destin du steamer est, à terme, de s'avouer vaincu, pour se retrouver sous la forme d'une épave (wreck) rouillant au bord du fleuve (fig. 3). Ce motif apparaît associé, chez Conrad déjà, à celui de la carcasse d'un mammifère mort, les côtes du squelette évoquant les membrures du bateau pourrissant, ou, ailleurs, à la carapace d'insectes non moins morts; il va se retrouver exemplairement chez Coppola sous la forme des avions militaires abattus, et en abondance dans les représentations $\mathrm{du}$ Congo contemporain, qu'elle soient verbales ou non-verbales, fictionnelles ou documentaires, y compris d'ailleurs sous la plume d'auteurs congolais, qui ne sont pas les derniers à désespérer de la situation ${ }^{23}$. 


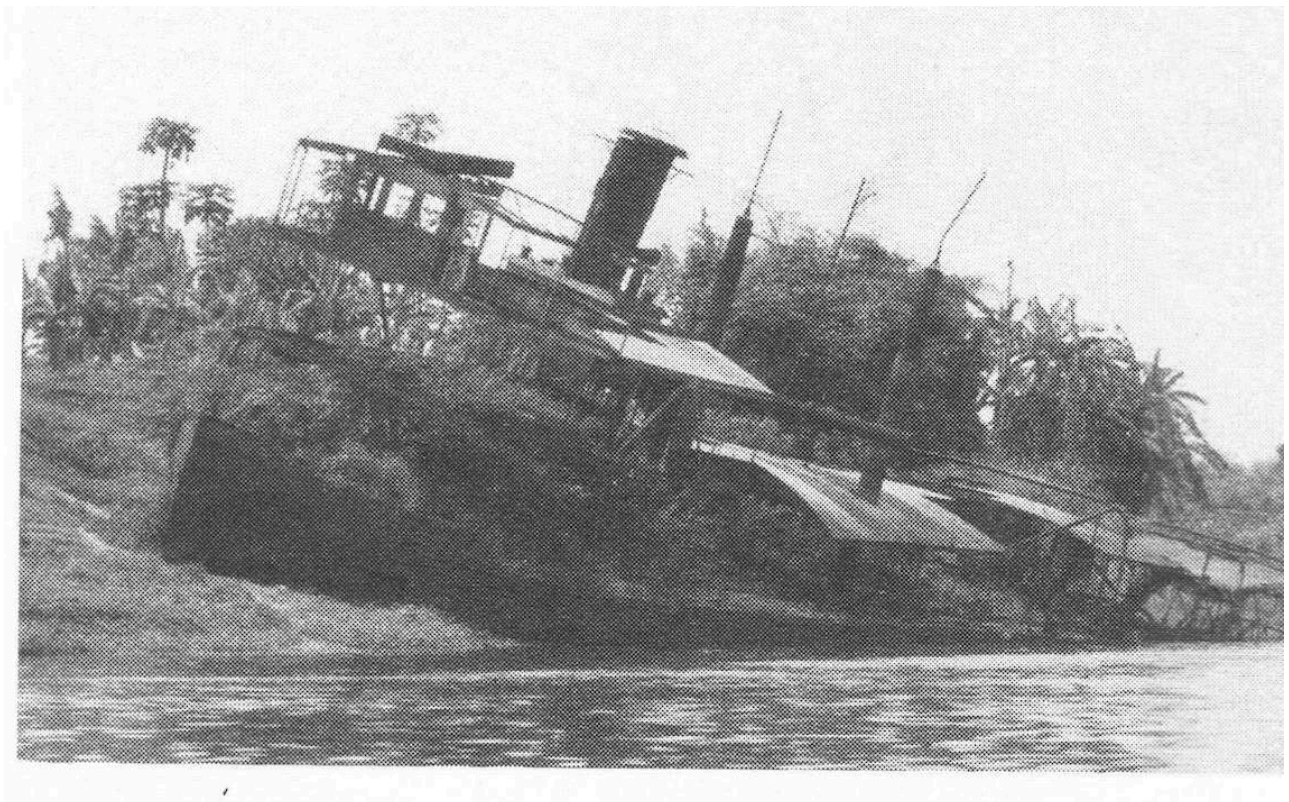

\section{Abandoned paddle-steamer, upper Congo River, August 2004}

Tim Butcher, Blood River : A Journey to Africa's broken Heart, London, Vintage, 2008, 363 p. ; p. 178. aquatiques est, à l'époque médiévale, le symbole très répandu de la communauté humaine et du peuple de Dieu que protège Marie, la Stella maris, on comprend peut-être encore mieux la place majeure qu'occupent, dans les documentaires récents consacrés au Congo, les barges surpeuplées et à maints égards « informelles ", poussées vaille que vaille par les remorqueurs qui ont pris la place des anciens steamers pour remonter le fleuve. Une variante du même discours, que nous ne pouvons développer ici faute de place, est le stade ultérieur de l'anti-progrès: la pirogue, et mieux, la pirogue représentée non pas dans un mouvement « en avant » comme on en voit fréquemment chez Stanley, mais immobile, voire, souvent, barrant même horizontalement un fleuve qui ne va lui-même plus nulle part, dont on ne voit pas le bout ou dont on est fasciné par les « chutes » : ce n'est pas la pirogue qui nous délivrera de la mélancolie.

Pour prendre la mesure de cette tradition conradienne par contraste, il nous faudrait également montrer ce qu'il en est de ce contre quoi elle s'est construite et ce contre quoi elle s'érige encore: un régime discursif «non conradien». On trouve celui-ci, notamment, sous la plume de Stanley, figure de fondateur admirée par le discours colonial ; sa statue fut ensuite déboulonnée autant que possible par l'anti-colonialisme, non seulement en raison de son rôle historique dans la colonisation, mais peut-être encore davantage en raison de signifiés seconds, relatifs aux brutalités du personnage, et en proportion de la gloire même qu'on lui avait prêtée jusque-là. Mais ici encore, si l'on dépasse cette couche idéologique dont la discussion est certes justifiée en soi, on trouve une sémiologie plus profonde, impliquant à la fois des valeurs et un langage : ils sont construits à l'opposé de ceux de Conrad, et pour cause, puisqu'ils sont structurés par l'idée que le progress est à la fois possible et nécessaire, qu'il est le «sens de l'Histoire ». Pour la tradition conradienne, ce sens dont on n'a plus même l'idée est l'objet perdu, perte abominable et désolante, insupportable souvent: de même que 
Marlow n'aura pas le courage d'infliger la vérité à la fiancée de Kurtz, de même les recycleurs «bien-pensants " de Coeur de ténèbres préfèreront en surligner la couche potentiellement militante qui, elle du moins, fait sens.

31 Comment, dans le discours contemporain, ces deux traditions à la fois sémiologiques et idéologiques entrent en belligérance, mais parfois aussi travaillent à se réconcilier, il serait possible également de le montrer, notamment dans la bande dessinée, secteur culturel de confluence entre publics divers, où se fabriquent sans doute des mémoires nouvelles ${ }^{24}$. Ce sont des essais de dépassements au sens fort, ou, à tout le moins, d'accommodement entre deux traditions discursives dont on aura compris que leur objet, le Congo, en cache souvent un autre, le sujet humain lui-même.

Figure 4. Couverture d'une édition illustrée récente de Cœur des ténèbres, publiée sous le double label de Gallimard et de Futuropolis

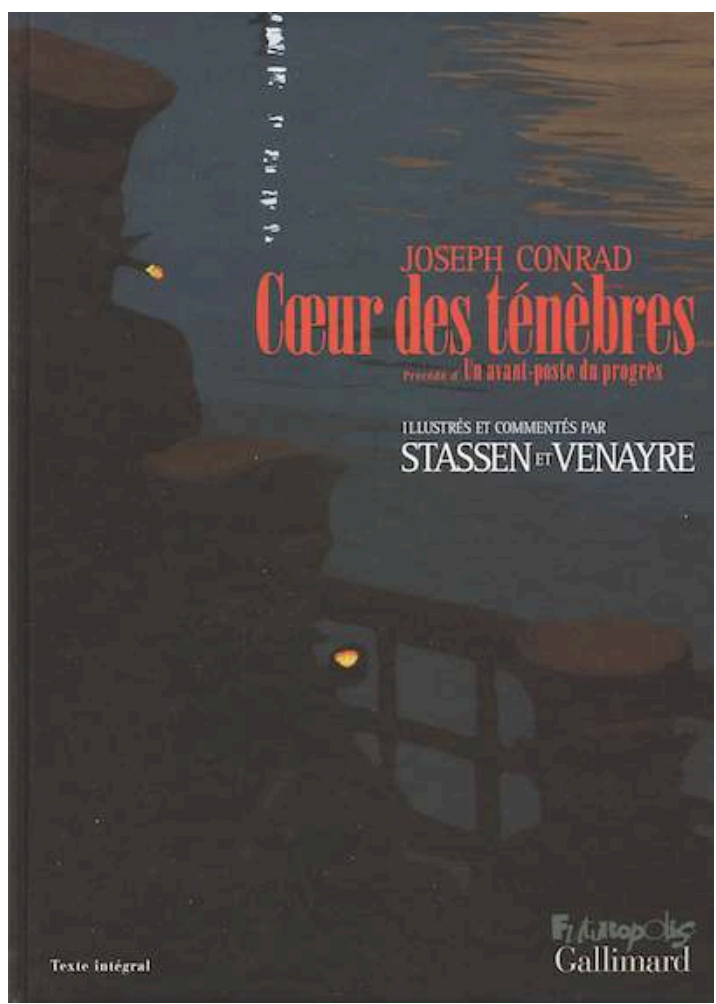

Le graphisme de Corto Maltese semble bien avoir inspiré la silhouette de Marlow vu par Stassen, ou en tout cas converger avec elle dans un même imaginaire viatique.

Joseph Conrad, Cœur des ténèbres, précédé d'Un avant-poste du progrès, traduits par Georges JeanAubry et André Ruyters, illustrés par Jean-Philippe Stassen et commentés par Sylvain Venayre (Introduction), Paris, Gallimard, coll. « Futuropolis », 2006, 139 p. ; 26,5 cm, ill.

\section{NOTES}

1. Du côté européen - en dehors des courants tiers-mondistes dont la confiance dans le «progrès » des «jeunes nations » est longtemps restée inébranlable en dépit de ce que d'autres 
observateurs qualifient fréquemment de «chaos congolais »-, la conversion des drames et des désordres observables est très rapide, qui passent du statut de difficultés transitoires à celui de « ténèbres », c'est-à-dire de manifestations d'une sauvagerie essentielle, liée au lieu ou non. C'est ce que montre, entre autres, pour la presse belge à l'époque de la «crise congolaise ", l'ouvrage de Bernard Piniau, auquel on doit une première approche diachronique de la tradition littéraire conradienne; cf. Bernard Piniau, Congo-Zaïre (1874-1981): la perception du lointain, Paris, L'Harmattan, coll. « Racines du Présent », 1992, p. 285.

2. Cf., entre autres, Pierre Halen, « De l'inusable imagerie du Cœur des ténèbres et de sa résurgence dans quelques représentations du génocide au Rwanda", dans Isaac Bazié et Hans-Jürgen Lüsebrink éd., Violences postcoloniales : représentations littéraires et perceptions médiatiques, Münster, LIT Verlag, coll. «Frankophone Literaturen und Kulturen außerhalb Europas », Bd. 4, 2011, 296 S. ; p. 65-88.

3. Voir notamment Pierre Halen, «L'Ouvert et le Fermé : une typologie de l'espace centreafricain dans toutes sortes d'histoires européennes ", dans Léonardy Ernst et Roland Hubert éd., Descriptions et créations d'espaces dans la littérature, Bruxelles, Nauwelaerts, 1995, p. 215-233; en ligne : https://www.academia.edu/11676898/; Id., « Stanley and Conrad, founders of Alternative Discourses about Central Africa», dans Elisabeth Mudimbe-Boyi éd., Remembering Africa, Portsmouth (NH), Heinemann, 2002, p. 56-73 ; Id., « De quelques enjeux du paysage (ou du nonpaysage) », Études littéraires africaines, $\mathrm{n}^{\circ}$ 39, Metz, Apela, juin 2015, p. 119-131 ; en ligne : http:// id.erudit.org/iderudit/1033136ar

4. Voir notamment Maëline Le Lay et al. éd., Archive (re)mix: vues d'Afrique, Rennes, Presses universitaires de Rennes, 2015, $242 \mathrm{p}$.

5. Cf. entre autres Jean-Luc Vellut, «La violence armée dans l'EIC: ténèbres et clartés de l'histoire d'un État conquérant ", Cultures et développement, 1984, t. 16, n 3-4, p.671-705; et plusieurs contributions dans J.-L. Vellut dir., La Mémoire du Congo : le temps colonial, Tervuren, Musée Royal de l'Afrique centrale, 2005, 271 p.

6. Voir notamment Pierre Halen, "Exotisme et antexotisme: notes sur les écrivains antiesclavagistes en Belgique francophone (1856-1894)", dans Papier blanc, encre noire : cent ans de culture francophone en Afrique centrale (Zaïre, Rwanda et Burundi), Émile Van Balberghe et al. éd., Bruxelles, Labor, coll. « Archives du Futur », 1992, 2 vol. , xcIV-690 p., ill. ; t. I, p. 35-53. En ligne : http://mukanda.univ-lorraine.fr/biblio/halen-pierre-exotisme-et-antexotisme-notes-sur-lesecrivains-antiesclavagistes-en-belgique-0

7. Red Rubber, livret et mise en scène de Alex Steyermark, musique de Dirk D’Haese, Anvers, théâtre De Singel, mai 1993 ; Africa Dreams, scénario : Maryse et Jean-François Charles, dessin : Frédéric Bihel, [Bruxelles-Paris], Casterman, 2010-2019, 4 vol.

8. Attente qui est sans doute en même temps d'un autre ordre : générationnel, identitaire ou, dirions-nous plus vaguement et faute de mieux, «civilisationnel » (ce qu'illustrait déjà, pour l'opinion belge de 1960, Bernard Piniau dans La Perception du lointain, op. cit.).

9. Ce n'est pas le cas dans An Outpost of progress, l'autre nouvelle inspirée à Conrad par son séjour congolais, dont les deux protagonistes s'appellent Kayerts - légère déformation d'un patronyme qui s'écrirait usuellement Keyaerts - et Carlier, deux patronymes qui renvoient de manière vraisemblable à deux identités flamande et wallonne.

10. https://www.littre.org/definition/tradition - c.09.06.2018

11. Il a connu un premier succès avec Youth. Il faudra néanmoins trois ans pour que le recueil Youth: A Narrative, and two other stories soit publié : l'éditeur Blackwood \& Sons (ce nom est tout fait pour susciter l'intérêt de Jean-Loup Cornille) n'est pas pressé ou n'a sans doute pas pensé qu'il ferait fortune en éditant ce livre.

12. Notamment du côté de l'enclave de Lado dont le sort ne sera finalement réglé qu'en 1910.

13. Cf. Chinua Achebe, "An Image of Africa : Racism in Conrad's Heart of Darkness ", Massachussets Review, $\mathrm{n}^{\circ}$ 18, 1977, p. 782-794. 
14. Ce qui est encore davantage le cas du film Posto-Avançado do Progresso / Un avant-poste du progrès Hugo Vieira da Silva (2017).

15. Un seul exemple : «À l'heure présente, l'ingénieur forge les derniers rails qui, du Cap Horn aux glaciers de l'Alaska, et des mers d'Extrême-Orient au Cap de Bonne-Espérance, doivent former l'anneau des épousailles de l'homme et de la terre. Par la colonisation, la jeunesse aventureuse décuple les champs ouverts à son activité, la lutte pour la vie se transforme en expansion nationale, le prolétaire fonde une famille, le convict redevient un citoyen, le déclassé se relève, l'individualisme triomphe du collectivisme, la richesse se féconde, le monde s'unifie, les préjugés s'effacent, la tolérance entre dans les cœurs et graduellement le sauvage cannibale, l'esclave héréditaire s'élève, grâce à d'augustes et généreuses initiatives, au niveau des races dominantes" (clausule des Légendes de l'inconnu géographique, par le comte G. de Lichtervelde, ancien ministre de Belgique aux États-Unis, Bruxelles, P. Lacomblez, 1903, 81 p.)

16. Silvia Riva, "La rhétorique des ténèbres dans Cannibale de Bolya Baenga ", dans L'Eil de l'autre, Tshibola Kalengayi et al. éd., Bruxelles, Archives et Musée de la Littérature; Kinshasa : CELIBECO, 2000, vol. B, p. 551-570.

17. Elle n'est pas moins présente dans le secteur de la large production exotisante comme le roman Congo (1980) de Michael Crighton, l'auteur de Jurassic Parc. Son rôle y est néanmoins différent : ainsi, dans le récit d'aventures, les thématiques conradiennes sont là avant tout pour augmenter le potentiel de résistance exotique que le lieu oppose au protagoniste européen, lequel doit triompher de toute l'altérité mais seulement, à vrai dire, pour s'en sortir vivant (comme Marlowe ou Tintin, ces deux évacués), et non pour en avoir réellement raison, ce qui n'arrive que dans le discours colonial et ses avatars post-coloniaux progressistes, littérairement moins intéressants, et pour cause.

18. Luc Rasson, “'Chacun sa place' : l'anticolonialisme dans Heart of Darkness (1899) et dans Voyage au bout de la nuit (1932) », dans Alain Buisine et al. éd., L'Exotisme, Paris, Diffusion Didier-Érudition, 1988, 468 p. ; p. 267-280.

19. Personnage qu'a développé, récemment, le roman de Marie Darrieussecq, Il faut beaucoup aimer les hommes, Paris, P.O.L, 2013.

20. "I've come to an end", dit le personnage de Querry (dont le nom semble venir en droite ligne du latin quarěre, «chercher ») : un personnage qui est de toute évidence "à bout », comme ne manque pas de le mettre en valeur la traductrice vers le français, Marcelle Sibon.

21. Voir par exemple : «[...] L'énorme Congo coule à l'allure rapide et massive des piétons à une heure de pointe sur les grands ponts de New York. Rien n'a changé ici depuis l'époque de Conrad. "Un cours d'eau désert, un grand silence, une forêt impénétrable". À perte de vue les petites îles d'herbe voguent vers la mer qu'elles n'atteindront jamais [...] Deux bateaux métalliques en train de rouiller. Nénuphars bleus. [...] »- Graham Greene, À la recherche d'un personnage : deux journaux africains, traduit de l'anglais par Marcelle Sibon, Paris, Robert Laffont, coll. « Pavillons », 1961, 188 p. ; p. 24-25.

22. Ténèbres liées, rappelons-le, à la mort du Christ sur la croix : «It was now about noon, and darkness came over the whole land " (Luc 23, 44).

23. Cf. Pierre Halen, «L'enfant-soldat vu par la fiction africaine : à propos de La Guerre et la Paix de Moni-Mambu par André Lye Yoka », Études littéraires africaines, n³2, déc. 2011, p. 91-105. En ligne : http://www.erudit.org/revue/ela/2011/v/n32/1018646ar.pdf

24. Pierre Halen, «Le Congo revisité. Une décennie de bandes dessinées belges (1982-1992)», Textyles, n 9, 1992, p. 365-382. En ligne : https://journals.openedition.org/textyles/2047 


\section{RÉSUMÉS}

Cœur des ténèbres, œuvre à la fois britannique et très internationale, a nourri la tradition conradienne, constituée d'éléments narratifs et thématiques qui lui ont été empruntés; dans le roman, les médias d'information ou les documentaires, dans la bande dessinée et au cinéma. Certaines de ces composantes sont des invariants d'ordre narratif, d'autres sont relatifs à l'espace, enfin il y a des thèmes plus abstraits comme celui du Diable. Le motif central du steamer, symbole du progrès, les cristallise et permet d'en lire les enjeux: au-delà de sa récupération idéologique toujours possible dans le sens du militantisme anti-colonialiste, la nouvelle apparaît ainsi comme une sorte de parabole civilisationnelle dont le cœur est l'« horreur » de l'Histoire elle-même.

Heart of Darkness, a both british and international work, forms the basis of the "conradian tradition", consisting of regrowth of thematic and narrative elements originating from the initial story. In novels, media, documentaries, comics or movies, those components can be narrative or narratologic invariants, patterns related to space and landscape, or more abstracts topics as the Evil. The steamer, central symbol of progress, condense them and let appear what's at stake: beyond the possible ideological appropriation by the anticolonialist discourse, Conrad's story can be seen as a kind of modern parable about civilisation, whose heart is the "horror" of the History self.

\section{INDEX}

Mots-clés : Joseph Conrad, Heart of Darkness, Archive, Idéologie, Anti-colonialisme, Progrès

\section{AUTEUR}

\section{PIERRE HALEN}

Pierre Halen est professeur à l'Université de Lorraine, directeur du Centre de recherche Écritures, directeur de la revue Études littéraires africaines. Ses domaines de recherche comprennent la littérature africaine, ainsi que la littérature et l'interculturalité. Pierre Halen est un des meilleurs connaisseurs de la littérature coloniale et post-coloniale belge, mais aussi de la littérature congolaise. À ce titre, il est l'auteur de très nombreuses publications en ouvrages collectifs, en revues ou de monographies telles que, pour n'en citer qu'une, Le petit Belge avait vu grand: une littérature coloniale (Labor, 1993). 